\title{
THEORETICAL AND EXPERIMENTAL STUDY ON VERTICAL DYNAMIC CHARACTERISTICS OF SIX-AXLE HEAVY-HAUL LOCOMOTIVE ON CURVE
}

\author{
Peng-Fei Liu ${ }^{1,2}$, Wan-Ming Zhai ${ }^{2}$, Kai-Yun Wang ${ }^{3}$, Quan-Bao Feng ${ }^{4}$, Zai-Gang Chen ${ }^{5}$ \\ ${ }^{1}$ School of Mechanical Engineering, Shijiazhuang Tiedao University, China \\ 2,3,5 State Key Laboratory of Traction Power, Southwest Jiaotong University, China \\ ${ }^{4}$ Beijing Feb. 7th Railway Transportation Equipment Co. Ltd., China
}

Submitted 24 September 2014; resubmitted 31 December 2014; accepted 20 April 2015; published online 04 May 2016

\begin{abstract}
This paper presents a method to study the vertical dynamic characteristics of a heavy-haul locomotive in curve. A quasi-static analysis model based on the static force equilibrium relationship is established to investigate the load bearing characteristics of suspension system when the locomotive runs through the curve. Then a locomotivetrack coupled dynamics model is used to analyse the dynamic characteristics of wheel load in curves. Finally, a field test in curve is carried out to validate the simulated results. The theoretical analysis results indicate that due to the different twist shapes of track on the entry and exit transition curves, for some specific position in the suspension system or wheel arrangements, the corresponding vertical load along the curve length presents an asymmetry about the section of circular curve. The asymmetry is predominantly caused by the Superelevation Angle Differences (SADs) between car body, bogie frames and wheelsets. A distinct phenomenon is that the outer wheel-rail vertical load of the first axle increases when the locomotive enters the transition curve and then reduces when it exits. These results are expected to provide theoretical guidance to the design of the heavy-haul railways. It is suggested that the asymmetric characteristics of the wheel loads can be improved by some measures, such as adopting a low vertical stiffness in the secondary suspension and increasing the transition curve length.
\end{abstract}

Keywords: locomotive; transition curve; suspension system; wheel load; vehicle-track coupled dynamics.

\section{Introduction}

Suspension system is one of the important components in a locomotive, which enables the improvement in the riding quality, the reduction of the impact on the wheelrail contact surface, and enhancement of the traction performance and so on. Locomotive motion postures will change accordingly to accommodate the line conditions, especially on a curved track under the action of the centrifugal force. The asymmetric load bearing in the suspension system may be caused easily, leading to the wheel load difference between the inner wheel and the outer wheel. It is a potential threat to the running safety of locomotives. In order to ensure the curving performance of vehicles, the circular curve and tangent track are generally connected with a transition curve where both the curve radius and superelevation vary continuously. The track plane presents a typical distorted shape in the sections of transition curves. With a larger variation gradient of the superelevation and curvature, the track plane twists more seriously, which has an impor- tant influence on the operation safety of railway vehicles ( $\mathrm{Gu}$ 2007). The running safety problems of vehicles caused by the track twist have long been focused on.

Wilson et al. (2011) introduced the assessment methods of railway vehicle running safety, including the simulation approach and field experimental technique. Some railway standards such as GM/RT 2141:2000, AS/RISSB 7509.1:2009 and BS EN 14363:2005, provide the track twist conditions, experimental methods and calculation methods to determine the vehicle's adaptability to the railway lines. However, there are still no relevant standards proposed aiming at the track twist phenomenon in China. The dynamic influence of the track twist on the vehicles in transition curves has not deserved concern.

As is known, a large number of studies on the dynamic performance of vehicles passing through the transition curve have been performed worldwide, which mainly focus on two aspects. One is to investigate the influences of vehicle structural parameters on vehicle 
system dynamics. Polach et al. (2006) evaluated vehicle-curving behaviour by computer simulation and put forward some techniques to improve the wheelset steering ability. Sinokrot et al. (2008) used a velocity transformation method to analyse the vehicle motions and wheel-rail forces in curves. Suarez et al. (2013) assessed the influence of the elastic properties of rail vehicle suspensions on its dynamic performance, and pointed out that the primary longitudinal stiffness was the most sensitive parameter. Another aspect is to investigate the effects of the line type and relevant parameter settings in transition curves on the evaluation indices of the vehicle dynamics. Eom and Lee (2010) analysed the influence of transition curve length on the curving performance of a diesel locomotive negotiating a curve with radius of $1200 \mathrm{~m}$, which indicated that its length affected the wheel load reduction slightly. Based on the comparisons of the vehicle system dynamic responses for different transition curves, Long et al. (2010) and Zhang et al. (2010) gave some suggestions to the railway curve design. For the conventional railway lines, Gailiene (2012) analysed the calculation defects of curve superelevation. It is explained clearly that more precise methods for calculating superelevation, evaluating actual train speed and considering permissible uncompensated lateral acceleration in the curves should be found. Um et al. (2011) developed a method to optimize the alignment of horizontal curves in case of the superimposition of horizontal curve and vertical curve. Lipičnik (1998) discussed the functions of transition curves from aspects of safety, comfort and economic driving. Miyagaki et al. (2004) studied the effect of transition forms on the vehicle responses, and proposed a new transition curve which was applied on an existing curve. Most research has focused on improving the vehicle curving performance by analysing the effects of vehicle conditions, transition forms and curve geometrical parameters. However, for a heavy-haul locomotive passing through the entry and exit transition region in a small radius curve, few studies have been carried out on dynamic wheel load characteristics.

As the standard of BS EN 14363:2005 shows, the track twist conditions will lead to wheel load reduction. The typical twist track exists in the sections of entry and exit transition curves. Under the track twist conditions on transition curves, the outer wheel loads will not always increase. The wheel load reduction may deteriorate the running safety. Especially for the leading wheelset of a six-axle locomotive on curves, the lateral wheel-rail dynamic interaction of the outer wheel is generally more severe so that a reduction of wheel load may result in an increase of the derailment coefficient. It is a potential threat to the locomotive running safety. In order to have a good knowledge about the influence of transition curves on dynamic wheel loads, both of the detailed varying characteristics of vertical loads in suspension system and wheel-rail contact interface are investigated in this paper. The basic force analysis of a six-axle locomotive running on a curved track is analysed in Section 1, which is followed by Sections 2 and 3 concerning the investigation on the vertical static and dynamic charac- teristics of the suspension system and the wheel loads. Then, a field test in a curve is implemented to verify the theoretical analysis results in Section 4. Finally, conclusions are given out.

\section{Calculation of Suspension Forces for a Six-Axle Locomotive on Curve}

In order to reduce or balance the inertial centrifugal force acting on the vehicle in curves, the superelevation is designed between the outer and inner rails. The gradual changing features of the transition curve are reflected in the variations of superelevation and curve radius. The typical twisted plane of the curved track is shown in Fig. 1. The symbols $h$ and $R$ represent the superelevation and curve radius, respectively. $O_{c} X_{c} Y_{c} Z_{c}$, $O_{t i} X_{t i} Y_{t i} Z_{t i}$ and $O_{w j} X_{w j} Y_{w j} Z_{w j}$ denote the body frames of the car body, bogie frame and wheelset, which are fixed at their gravity centers, respectively. The three body frames follow the motions of their respective corresponding bodies. $\theta_{c}, \theta_{t 1}$ and $\theta_{t 2}$ are the superelevation angles of rails at the locations of the mass centers of the car body, the front bogie and the rear bogie. Generally, if the running velocity is not equal to the equilibrium speed for a certain superelevation, the outer and inner wheel loads of a wheelset will vary conversely (increase or reduce). Variations of the wheel loads are closely related with the line geometric features.

The dynamic wheel loads are dependent on the load transfer behaviours in the locomotive-track system. Significantly, the analysis of relative displacements in the suspension elements is the essential prerequisite to accurately solve the load distributions and transfer relationship. For the locomotive affected by the twisted track plane in curves, there are complex transformation relations between the body reference frames.

For the locomotive running on a transition curve, the suspension positions of the car body and bogie in the secondary suspension are denoted by $\left\{r_{c p}\right\},\left\{r_{t p}\right\}$, as shown in Fig. 2. The distance between the mass centers of the car body and the front bogie consists of three displacement components, namely the $b$ (longitudinal), $c$ (lateral) and $d$ (vertical). $\alpha$ is the included angle between $x$ axis of the car body frame and $x$ axis of the bogie body frame.

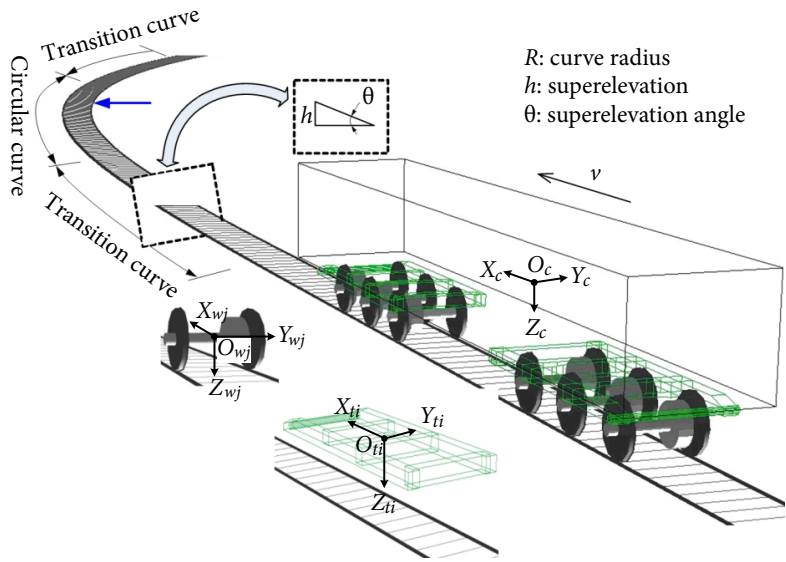

Fig. 1. Curved track 


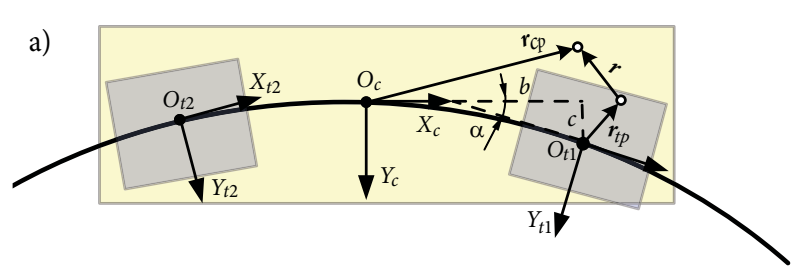

b)

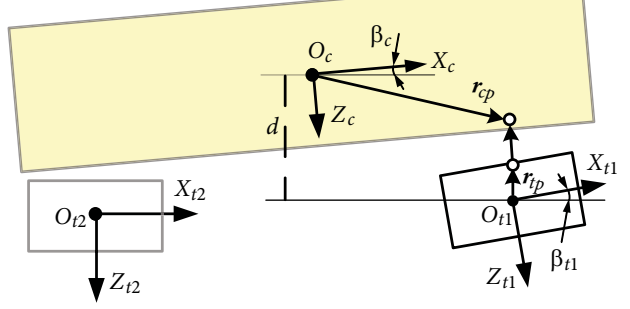

Fig. 2. Relative displacement at the secondary suspension points of vehicle in curve: $a$ - top view; $b$ - side view

According to Slivsgaard (1995) and Wang, Zhai (2003), the displacements of the secondary suspension points with respect to their respective inertial system are given by:

$$
\begin{aligned}
& \left\{\Delta r_{c p}\right\}=\left\{\Delta r_{c}\right\}+\left[A_{c p}\right]\left\{r_{c p}\right\}-\left\{r_{c p}\right\} ; \\
& \left\{\Delta r_{t p}\right\}=\left\{\Delta r_{t}\right\}+\left[A_{t p}\right]\left\{r_{t p}\right\}-\left\{r_{t p}\right\},
\end{aligned}
$$

where: $\left[A_{c p}\right],\left[A_{t p}\right]$ are the transform matrices delivering the coordinates in the initial systems of the car body and the bogie to that in the body frames; $\left\{\Delta r_{c}\right\}$, $\left\{\Delta r_{t}\right\}$ represent the translation vectors of the car body and bogie body frames, respectively.

Choosing the body frame of car body as the basic reference frame, the secondary suspension forces are calculated. Taking the front bogie as an example, the transform relation from the bogie body frame to car body coordinates are expressed as:

$$
\begin{aligned}
& \left\{\begin{array}{l}
X_{c} \\
Y_{c} \\
Z_{c}
\end{array}\right\}=\left[\begin{array}{ccc}
1 & -\alpha & 0 \\
\alpha & 1 & -\left(\theta_{t 1}-\theta_{c}\right) \\
0 & \theta_{t 1}-\theta_{c} & 1
\end{array}\right]\left\{\begin{array}{l}
X_{t 1} \\
Y_{t 1} \\
Z_{t 1}
\end{array}\right\}+\left\{\begin{array}{l}
b \\
c \\
d
\end{array}\right\}= \\
& {\left[\begin{array}{l}
A_{c t}
\end{array}\right]\left\{\begin{array}{lll}
X_{t 1} & Y_{t 1} & \left.Z_{t 1}\right\}^{T}+\left\{r_{0}\right\} .
\end{array}\right.}
\end{aligned}
$$

The relative displacement at the secondary suspension point of the front bogie can be determined as:

$$
\{\Delta s\}=\left\{\Delta r_{c p}\right\}-\left(\left[A_{c t}\right]\left\{\Delta r_{t p}\right\}+\left\{r_{0}\right\}\right)=\left\{\begin{array}{c}
\Delta s x L 1 \\
\Delta s y L 1 \\
\Delta s z L 1
\end{array}\right\},
$$

where: $\Delta_{s x L 1}, \Delta_{s y L 1}, \Delta_{s z L 1}$ are the components of $\{\Delta s\}$ in the longitudinal, lateral and vertical directions.

The Superelevation Angle Difference (SAD) between the locations corresponding to the wheelset and bogie frame mass center is defined as $\Delta \theta_{w j}$, and that between the bogie frame and car body mass centers is defined as $\Delta \theta_{t i}$, which can be calculated as:

$$
\left\{\begin{array}{l}
\Delta \theta_{t i}=\theta_{t i}-\theta_{c} \\
\Delta \theta_{w j}=\theta_{w j}-\theta_{t i}
\end{array}\right.
$$

where: the subscript $i(i=1,2)$ represents the front and rear bogie frame, respectively; the symbol $j(j=1 \ldots 6)$ represents the wheelset number (for $i=1 ; j=1,2,3$ and for $i=2 ; j=4,5,6)$. By simplifying the relative displacements, the outside and inside lateral relative displacements $\left(\Delta s_{y(L, R) i}\right)$ at the secondary suspension of front and rear bogies are expressed by:

$$
\begin{aligned}
& \Delta s y(L, R) i=y_{c}-y_{t i}-h_{b t} \phi_{t i}- \\
& h_{c b} \phi_{c}-(-1)^{i} l_{c} \psi_{c}-\frac{l_{c}^{2}}{2 R_{c}}
\end{aligned}
$$

and the vertical relative displacements are:

$$
\begin{aligned}
& \Delta s z(L, R) i=z_{c}-z_{t i}-(-1)^{i-1} l_{c} \beta_{c} \mp \\
& d_{s} \phi_{c} \pm d_{s} \phi_{t i} \pm \Delta \theta_{t i} d_{s}-\frac{h_{c}-h_{t i}}{2},
\end{aligned}
$$

where: $L, R$ represent the outer and inner suspension; the upper sign of both \pm and $\mp$ are selected for the outer suspension, and the lower sign for the inner suspension; $z_{c}, y_{c}$ denote the vertical and lateral displacements of the car body; $\beta_{c}, \psi_{c}, \phi_{c}$ represent the angles around $x, y$ and $z$ coordinate axis of the car body; $z_{t i}, y_{t i}$ are the vertical and lateral displacements of the bogie frame and $\phi_{t i}$ is its roll angle; $h_{c b}, h_{b t}$ are the distances from mass centers of the car body and bogie frame to the secondary suspension; $h_{c}, h_{t i}$ are the distances from mass centers of the car body and bogie frame to the track horizontal plane; $d_{s}$ is the half distance of secondary suspension in lateral direction, $l_{c}$ is the half of distance between bogie centers; $R_{c}$ is the curve radius at the car body center.

The vertical relative displacements in the primary suspension are expressed as:

$$
\left\{\begin{array}{l}
\Delta p_{z(L, R) j}=d_{1}, \quad j=1,3,4,6 ; \\
\Delta p_{z(L, R) j}=d_{2}, \quad j=2,5,
\end{array}\right.
$$

where:

$$
\begin{aligned}
& d_{1}=z_{t i}-z_{w j}+(-1)^{i} l_{t} \beta_{t i} \mp d_{s} \phi t_{i} \pm \\
& d_{w} \phi_{w j} \pm \Delta \theta_{w j} d_{w}-\frac{h_{t i}-h_{w j}}{2} \\
& d_{2}=z_{t i}-z_{w j} \mp d_{s} \phi t_{i} \pm \\
& d_{w} \phi_{w j} \pm \Delta \theta_{w j} d_{w}-\frac{h_{t i}-h_{w j}}{2}
\end{aligned}
$$

where: $z_{w j}$ is the vertical displacement of wheelset; $\beta_{t i}$ is the rotation angle of truck around $y$ axle; $l_{t}$ is the half of total wheelbase; $\phi_{w j}$ represent the wheelset roll angle; $h_{t i}$, $h_{\mathrm{wj}}$ are the distances from the mass centers of the bogie frame and wheelset to the track horizontal plane.

The vertical forces of the primary and secondary suspensions are calculated by:

$$
\begin{aligned}
& F_{s z(L, R) i}=k_{s z} \cdot \Delta s_{z(L, R) i} ; \\
& F_{p z(L, R) j}=k_{s z} \cdot \Delta p_{z(L, R) j,}
\end{aligned}
$$


where: $k_{s z}, k_{p z}$ denote the vertical stiffness in the primary and secondary suspensions.

It is indicated in Eqs (7-9) that the asymmetries between the outer and inner vertical loads in the primary and secondary suspensions are mainly contributed by $\phi_{c}$, $\phi_{t i}, \phi_{w j}, \Delta \theta_{w j}$ and $\Delta \theta_{t i}$.

\section{Load Characteristics of Suspension System Analysed with Quasi-Static Model}

\subsection{Quasi-Static Analysis Model of Load Bearing Behaviour}

In order to highlight the influence of track twist on vertical load distributions in the suspension system, the quasi-static analysis method is used. In this section, taking the car body and bogie frame as the research objects, the load bearing characteristics of the suspension are investigated based on the quasi-static analysis method. When the locomotive runs on a curved track with a deficient superelevation, it will generally be pushed to the outer rail side due to the effect of unbalanced inertial centrifugal force. The vertical force acting on the outer rail presents an increasing tendency. In the locomotive system, the car body takes the major mass of the entire system, which contributes the maximum inertial centrifugal force. The force will directly impact the lateral movement and rolling motion of car body. Undoubtedly, the variation of car body motion will lead to the asymmetrical load bearing in the suspension systems, and then has an effect on the wheel load reduction.

In curves, the lateral motion of the car body is generally accompanied with its rolling motion. In order to analyse the load bearing behaviour of suspension system in the vertical direction, a simplified model is established, in which it is assumed that the car body has the lateral and roll motions and the bogie frame can only rotate around its $x$ coordinate axis. The force conditions are shown in Fig. 3. $F_{g y}$ is the unbalanced centrifugal force; $M_{c}$ and $M_{t}$ are the masses of car body and bogie frame, respectively.

Based on Eqs (6-8), the corresponding suspension forces are calculated as:

$$
\left\{\begin{array}{l}
F_{y L i}=F_{y R i}=k_{s y}\left(\phi_{c} h_{c b}-y_{c}+\frac{l_{c}^{2}}{2 R_{c}}\right) \\
F_{s z(L, R) i}= \pm k_{s z}\left(\Delta \theta_{t i}-\phi_{c}+\phi_{t i}\right) d_{s}+\frac{M_{c} g}{4} \\
F_{p z(L, R) j}= \pm k_{p z}\left(\Delta \theta_{w j}-\phi_{t i}\right) d_{s}+\frac{M_{c} g}{12}+\frac{M_{t} g}{4},
\end{array}\right.
$$

where: $g$ is the gravity acceleration.

The unbalanced centrifugal force acting on the car body is denoted as:

$$
F_{g y}=M_{c} \frac{v^{2}}{R_{c}}-M_{c} g \sin \theta_{c} .
$$

Then, the equilibrium equations of the system can be obtained as:
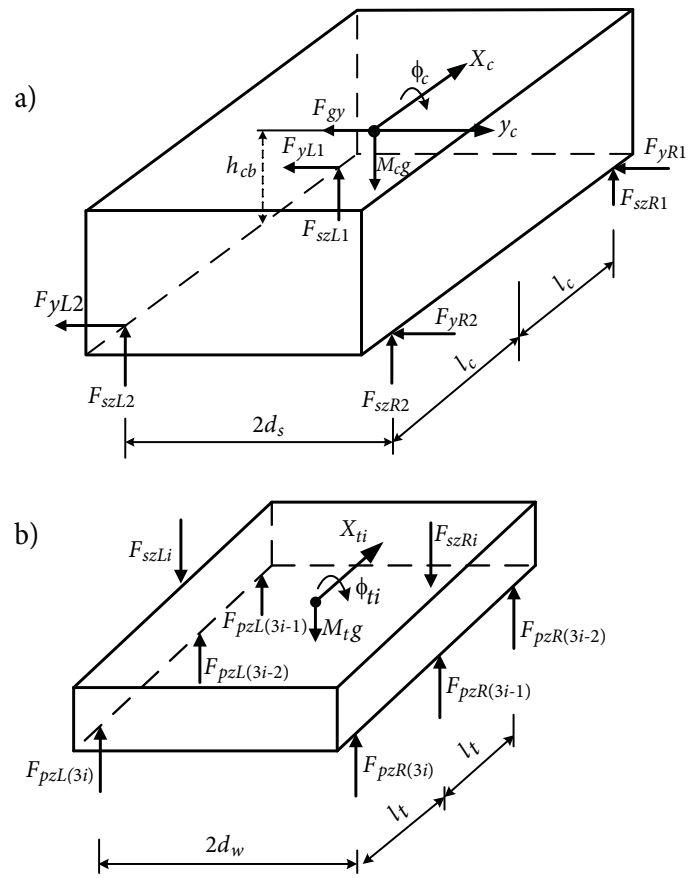

Fig. 3. Force conditions of the car body and bogie frame in the simplified model: $\mathrm{a}$ - car body; $\mathrm{b}$ - bogie frame

$$
\left\{\begin{array}{l}
F_{y L 1}+F_{y L 2}+F_{y R 1}+F_{y R 2}+F_{g y}=0 ; \\
\sum_{i=1}^{2}\left(F_{s z L i}-F_{s z R i}\right) d_{s}+\sum_{i=1}^{2}\left(F_{y L i}+F_{y R i}\right) h_{c b}=0 ; \\
\sum_{j=1}^{3}\left(F_{p z L j}-F_{p z R j}\right) d_{w}-\left(F_{s z L 1}-F_{s z R 1}\right) d_{s}=0 ; \\
\sum_{j=4}^{6}\left(F_{p z L j}-F_{p z R j}\right) d_{w}-\left(F_{s z L 2}-F_{s z R 2}\right) d_{s}=0 .
\end{array}\right.
$$

Eq. (12) is a system of linear equations, which can be easily solved. Finally, the roll angles of the car body and bogie frame can be obtained as:

$$
\begin{aligned}
& \phi_{c}=\frac{-h_{c b}}{12 k_{p z} k_{s z} d_{w}^{2} d_{s}^{2}}\left(k_{s z} d_{s}^{2}+3 k_{p z} d_{w}^{2}\right) \times \\
& \left(\frac{M_{c} v^{2}}{R_{c}}-M_{c} g \sin \theta_{c}\right)+ \\
& \frac{1}{6}\left(3 \sum_{i=1}^{2} \Delta \theta_{t i}+\sum_{j=1}^{6} \Delta \theta_{w j}\right) ; \\
& \phi_{t i}=\frac{k_{p z} d_{w}^{2} \sum_{j=3 i-2}^{3 i} \Delta \theta_{w j}+k_{s z} d_{s}^{2}\left(\phi_{c}-\Delta \theta_{t i}\right)}{3 k_{p z} d_{w}^{2}+k_{s z} d_{s}^{2}} .
\end{aligned}
$$

Substituting Eqs (13-14) into Eq. (10), the vertical forces in suspension system can be calculated. It is clear that the properties of vertical load distributions in the locomotive suspension system are closely related to the railway geometry parameters. 


\subsection{Analysis of Suspension Load Bearing Characteristics}

In order to increase the utilization factor of adhesive weight, numbers of locomotives are equipped with rubber elements in its secondary suspension, which can provide larger vertical stiffness. Taking a heavy-haul locomotive as an example, the load bearing characteristics of the suspension system on curved track are analysed. The basic parameters of locomotive, which will be used in the simplified model, are listed in Table 1. In the curve, its radius is $300 \mathrm{~m}$, the superelevation of outer rail is $120 \mathrm{~mm}$ and the length of transition curve is $70 \mathrm{~m}$. The superelevation and curvature in the transition curve section changes linearly along the track length. When the locomotive is running in the curve at a speed of $70 \mathrm{~km} / \mathrm{h}$, the superelevation is deficient.

Table 1. Design parameters of the locomotive

\begin{tabular}{|c|l|c|}
\hline Notation & \multicolumn{1}{|c|}{ Parameter } & Value \\
\hline$M_{c}$ & Car body mass [kg] & 88862 \\
\hline$M_{t}$ & Truck frame mass [kg] & 13500 \\
\hline$k_{s z}$ & $\begin{array}{l}\text { Vertical stiffness of secondary } \\
\text { suspension for one side of bogie } \\
{[\mathrm{MN} / \mathrm{m}]}\end{array}$ & 18 \\
\hline$k_{p z}$ & $\begin{array}{l}\text { Vertical stiffness of primary suspension } \\
\text { for each axle-box [MN/m] }\end{array}$ & 1.7 \\
\hline$d_{s}$ & $\begin{array}{l}\text { Half of lateral distance in secondary } \\
\text { suspension [m] }\end{array}$ & 1.1 \\
\hline$d_{w}$ & $\begin{array}{l}\text { Half of lateral distance in primary } \\
\text { suspension [m] }\end{array}$ & 1.0 \\
\hline$h_{c b}$ & $\begin{array}{l}\text { Distance from car body mass center } \\
\text { to secondary suspension [m] }\end{array}$ & 0.6 \\
\hline$l_{c}$ & Half of bogie center distance [m] & 6.16 \\
\hline$l_{t}$ & Half of bogie wheelbase [m] & 2.0 \\
\hline
\end{tabular}

The SADs of the front and rear bogie frames are shown in Fig. 4. It is indicated that as the whole locomotive system has entered into the transition curves, the SAD $\Delta \theta_{t i}$ doesn't change with the distance increasing. It is due to the assumption of the linear variation of superelevation in transition curves. In the regions near the

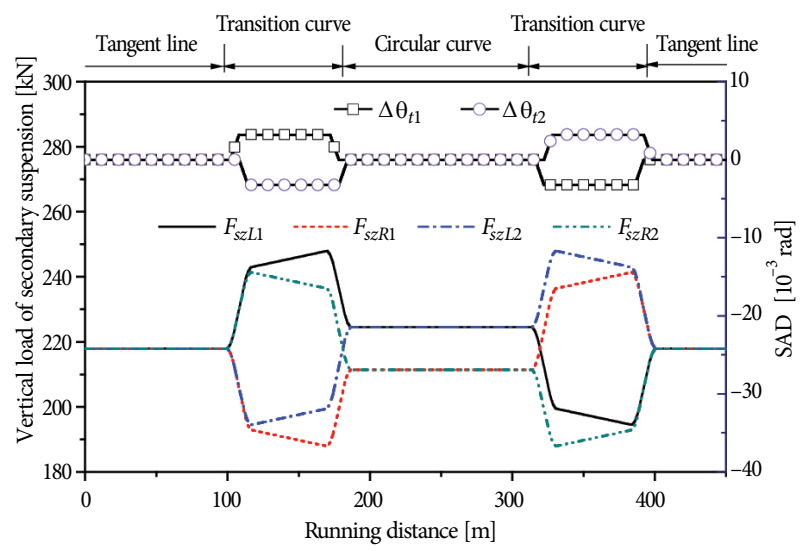

Fig. 4. Secondary suspension forces and SADs of bogie frames in curve connecting points of tangent to spiral, spiral to curve, curve to spiral and spiral to tangent, the car body and bogie frame don't run on the transition curve or circular curve simultaneously so that the value $\Delta \theta_{t i}$ varies distinctly within a short distance. The influences of $\Delta \theta_{t i}$ on the vertical forces of the secondary suspension are also shown in Fig. 4. It can be found that the outer and inner suspension forces for a certain bogie present an inverse changing law along the running distance. When the locomotive enters the transition curve, the SAD of front bogie $\left(\Delta \theta_{t 1}\right)$ is positive which leads to the compress of outer suspension and stretch of the inner suspension relative to the equilibrium position. As a result, the load of outer suspension increases and that of the inner suspension reduces. In the circular segment of the curve, the values of $\Delta \theta_{t 1}$ and $\Delta \theta_{t 2}$ are equal to zero and the vertical loads of suspension system are determined only by the roll angles of the car body and bogie frame. Under the action of unbalanced centrifugal force, the car body will roll toward outer rail, which causes the load increase in the outer suspension and load reduction in the inner suspension.

For the primary suspension loads $F_{p z(L, R) j}$ in Eq. (10), $M_{c} g$ and $M_{t} g$ are constants. The parameters, which affect these loads, are limited to $\Delta \theta_{w j}$ and $\phi_{t i}$. The sign and module of $\left(\Delta \theta_{w j}-\phi_{\mathrm{ti}}\right)$ reflect the dynamic variations of the primary suspension forces as well as the influence extent of the curve geometric parameters. Fig. 5 shows the SADs of wheelsets and roll angles of bogie frames.

At the entry of the transition curve, the vertical load of the outer secondary suspension increases. The front bogie frame has a negative roll angle and also the SAD at the first wheelset is positive (Fig. 5). For the first wheelset, both of the two factors make the outer spring of the primary suspension compressed to a larger degree, which results in the load increase. Conversely, the inner spring of the primary suspension is stretched, which leads to the load reduction. For the second wheelset, the value of SAD is close to zero and the load bearing characteristics are determined by the roll angle of bogie frame. As for the third wheelset, its SAD at the gravity center is negative which will offset a part of the vertical suspension force produced by the negative roll angle of bogie frame. At the exit transition curve, the influences of wheelset SADs on the suspension loads are contrary. In addition, the load bearing characteristics of the rear bogie suspension have an inverse property compared with that of the front bogie. The detailed bearing states of the primary suspension are listed in Table 2 .

Fig. 6 gives the calculated primary suspension loads in the curve. When the entire locomotive runs on the transition curves, the module $\left|\Delta \theta_{w j}-\phi_{t i}\right|$ agrees with Eqs (15-16). Among them, the values of $\left|\Delta \theta_{w 1}-\phi_{t 1}\right|$ and $\left|\Delta \theta_{w 6}-\phi_{t 2}\right|$ are maximum. It also can be found in Fig. 6 that the vertical forces of the primary suspension of the first and sixth wheelset are influenced most greatly, and they fluctuate drastically in the transition curve sections.

$$
\begin{aligned}
& \left|\theta_{w 1}-\phi_{t 1}\right|>\left|\theta_{w 2}-\phi_{t 1}\right|>\left|\theta_{w 3}-\phi_{t 1}\right| ; \\
& \left|\theta_{w 4}-\phi_{t 2}\right|<\left|\theta_{w 5}-\phi_{t 2}\right|<\left|\theta_{w 6}-\phi_{t 2}\right| .
\end{aligned}
$$


a)

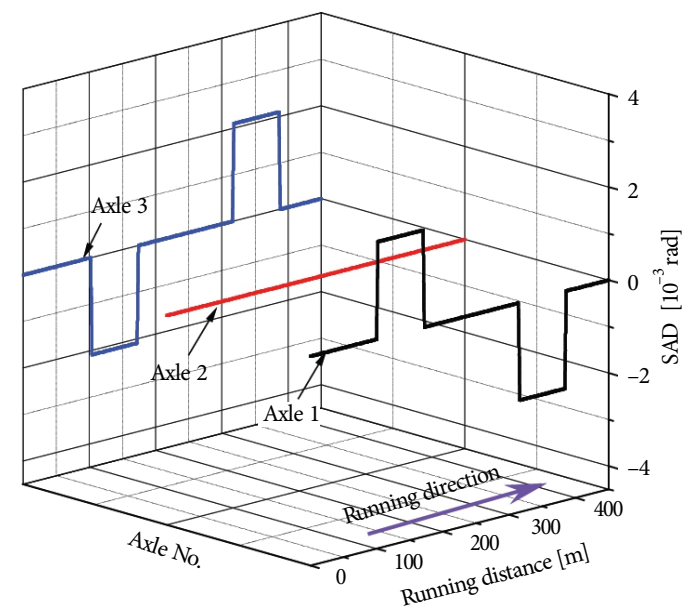

b)
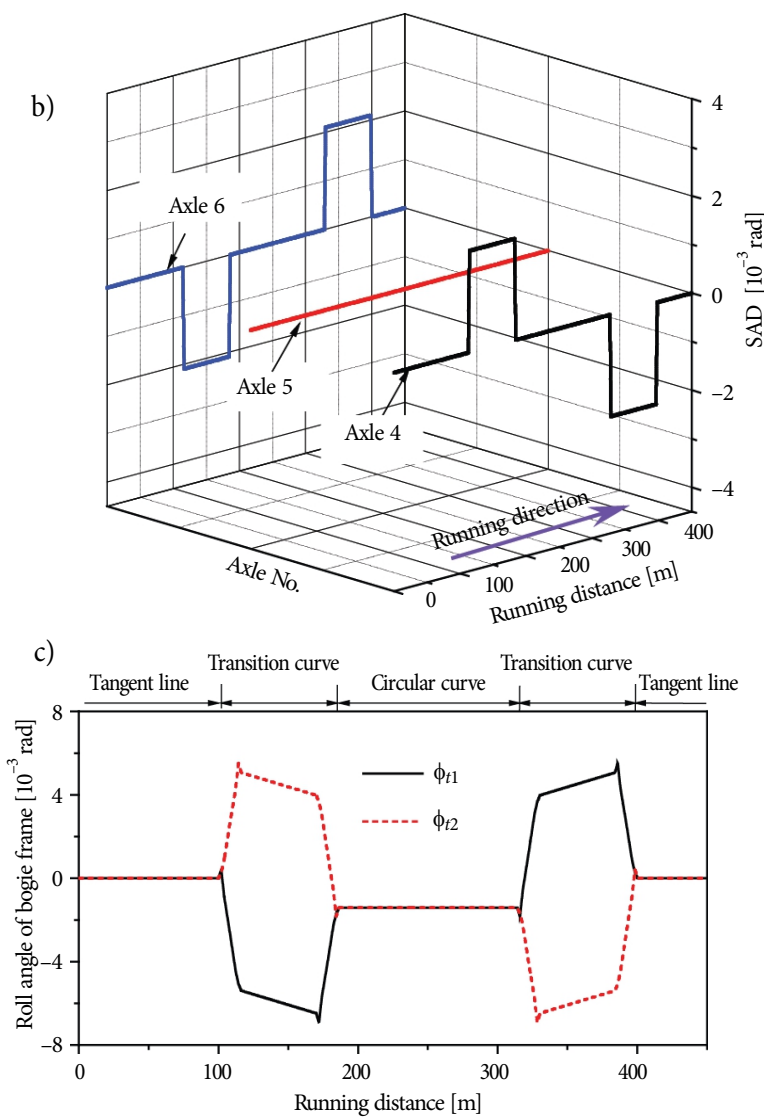

Fig. 5. SADs of wheelsets and roll angles of bogie frames: a - SADs of axle 1...3; b - SADs of axle 4...6; $\mathrm{c}$ - roll angles of front and rear bogie frames a)

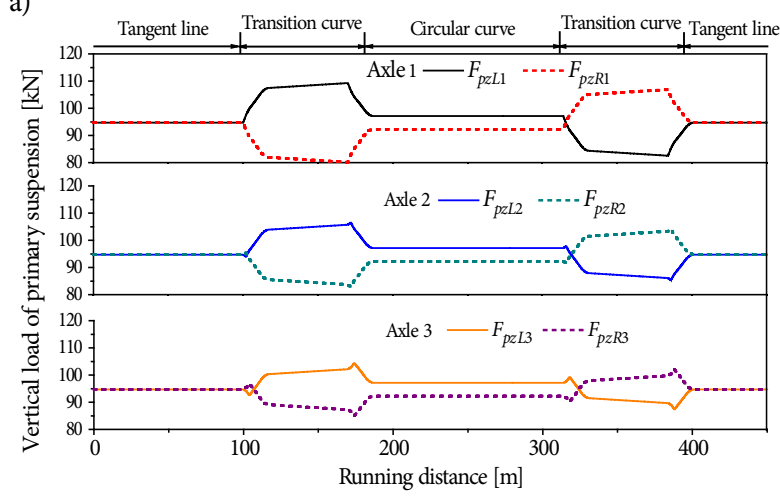

b)

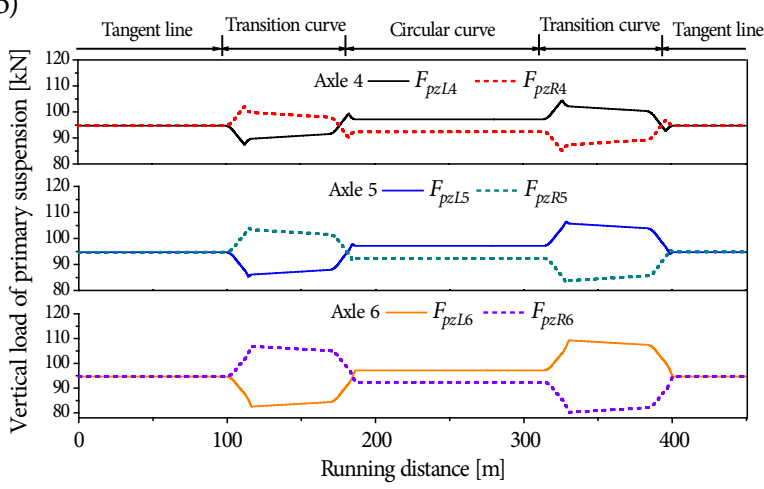

Fig. 6. Primary suspension forces considering SAD: $\mathrm{a}$ - front bogie; $\mathrm{b}$ - rear bogie

\section{Dynamic Characteristic of Wheel Load for Locomotive on Curve}

Based on the theory and modelling method of vehicletrack coupled dynamics (Zhai et al. 1996, 2009, 2013), a locomotive-track coupled dynamic model is established to study the dynamic characteristics of the wheel load in curves. The model consists of a locomotive and a track submodels, as shown in Fig. 7. In the locomotive model, every rigid body has five Degrees Of Freedom (DOF) with the stiffness and damping parameters. In the ballast track model, the rail is simplified as an Euler beam with vertical, lateral and torsional DOFs. In addition, the rail vibration is resolved with the modal superposition method. For the sleeper, it is regarded as a rigid body, which also has vertical, lateral and torsional DOFs. The ballasts are simulated as discrete rigid masses with ver-

Table 2. The load bearing characteristics of primary suspension on the entry and on the exit transition curve

\begin{tabular}{|c|c|c|c|c|c|c|c|}
\hline Item & Axle & 1 & 2 & 3 & 4 & 5 & 6 \\
\hline \multicolumn{8}{|c|}{ The load bearing characteristics of primary suspension on the entry transition curve } \\
\hline \multicolumn{2}{|c|}{ Variable information } & $\Delta \theta_{w 1}>0 ; \phi_{t 1}<0$ & $\Delta \theta_{w 2}=0 ; \phi_{t 1}<0$ & $\Delta \theta_{w 3}<0 ; \phi_{t 1}<0$ & $\Delta \theta_{w 4}>0 ; \phi_{t 2}>0$ & $\Delta \theta_{w 5}=0 ; \phi_{t 2}>0$ & $\Delta \theta_{w 6}<0 ; \phi_{t 2}>0$ \\
\hline \multirow{2}{*}{$\begin{array}{l}\text { Load } \\
\text { characteristic }\end{array}$} & outer & increase & increase & \multirow{2}{*}{$\begin{array}{c}\text { dependent on } \\
\operatorname{sign}\left(\Delta \theta_{w 3}-\phi_{t 1}\right)\end{array}$} & \multirow{2}{*}{$\begin{array}{c}\text { dependent on } \\
\operatorname{sign}\left(\Delta \theta_{w 4}-\phi_{t 2}\right)\end{array}$} & reduction & reduction \\
\hline & inner & reduction & reduction & & & increase & increase \\
\hline \multicolumn{8}{|c|}{ The load bearing characteristics of primary suspension on the exit transition curve } \\
\hline \multicolumn{2}{|c|}{ Variable information } & $\Delta \theta_{w 1}<0 ; \phi_{t 1}>0$ & $\Delta \theta_{w 2}=0 ; \phi_{t 1}>0$ & $\Delta \theta_{w 3}>0 ; \phi_{t 1}>0$ & $\Delta \theta_{w 4}<0 ; \phi_{t 2}<0$ & $\Delta \theta_{w 5}=0 ; \phi_{t 2}<0$ & $\Delta \theta_{w 6}>0 ; \phi_{t 2}<0$ \\
\hline \multirow{2}{*}{$\begin{array}{l}\text { Load } \\
\text { characteristic }\end{array}$} & outer & reduction & reduction & \multirow{2}{*}{$\begin{array}{c}\text { dependent on } \\
\operatorname{sign}\left(\Delta \theta_{w 3}-\phi_{t 1}\right)\end{array}$} & \multirow{2}{*}{$\begin{array}{c}\text { dependent on } \\
\operatorname{sign}\left(\Delta \theta_{w 4}-\phi_{t 2}\right)\end{array}$} & increase & increase \\
\hline & inner & increase & increase & & & reduction & reduction \\
\hline
\end{tabular}




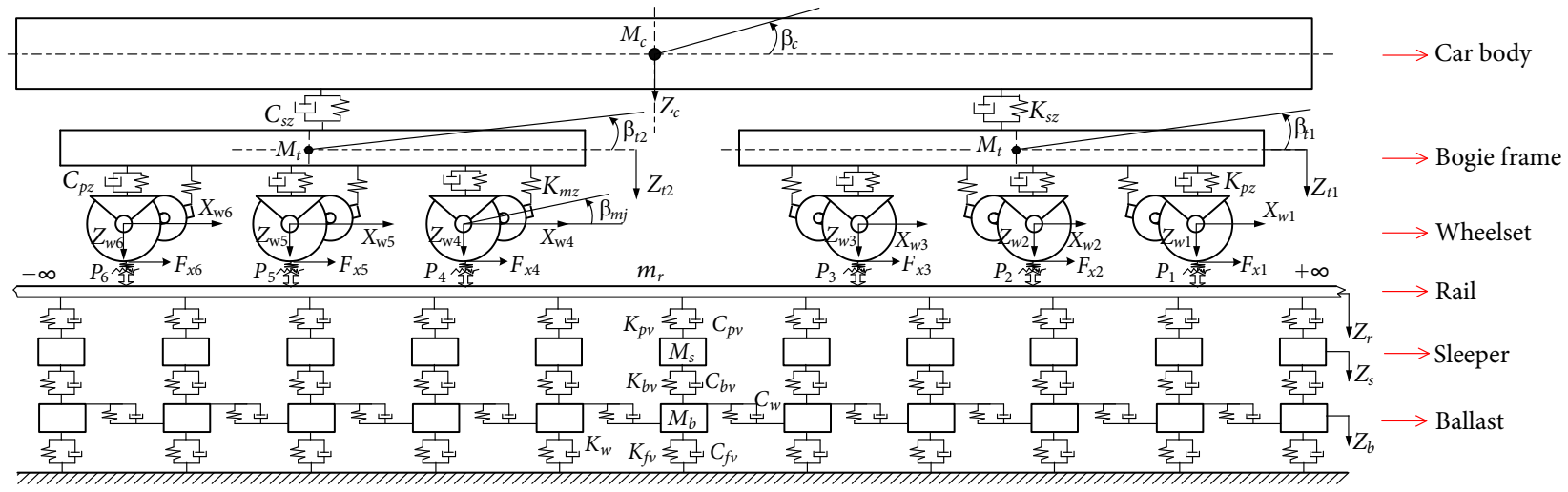

Fig. 7. Locomotive-track coupled dynamics model (side view)

tical DOF, and it is supported by the subgrade stiffness and damping. For the existing railway line, the track parameters used in this paper are listed in Table 3.

Table 3. Main parameters of track used in simulation

\begin{tabular}{|c|l|c|}
\hline Notation & \multicolumn{1}{|c|}{ Parameter } & Value \\
\hline$m_{r}$ & Rail mass per unit length $[\mathrm{kg} / \mathrm{m}]$ & 60.64 \\
\hline$M_{s}$ & Sleeper mass [kg] & 237 \\
\hline$M_{b}$ & Ballast discrete mass [kg] & 682.6 \\
\hline$K_{p v}$ & $\begin{array}{l}\text { Fastener stiffness in vertical direction } \\
{[\mathrm{N} / \mathrm{m}]}\end{array}$ & $7.8 \cdot 10^{7}$ \\
\hline$C_{p v}$ & $\begin{array}{l}\text { Fastener damping in vertical direction } \\
{[\mathrm{N} / \mathrm{m}]}\end{array}$ & $5.0 \cdot 10^{4}$ \\
\hline$K_{b v}$ & Ballast vertical stiffness $[\mathrm{N} / \mathrm{m}]$ & $2.4 \cdot 10^{8}$ \\
\hline$C_{b v}$ & Ballast vertical damping $[\mathrm{N} \cdot \mathrm{s} / \mathrm{m}]$ & $5.88 \cdot 10^{4}$ \\
\hline$K_{w}$ & Ballast shear stiffness $[\mathrm{N} / \mathrm{m}]$ & $7.8 \cdot 10^{7}$ \\
\hline$C_{w}$ & Ballast shear damping $[\mathrm{N} \cdot \mathrm{s} / \mathrm{m}]$ & $8.0 \cdot 10^{4}$ \\
\hline$K_{w}$ & Subgrade stiffness $[\mathrm{N} / \mathrm{m}]$ & $6.5 \cdot 10^{7}$ \\
\hline$C_{w}$ & Subgrade damping $[\mathrm{N} \cdot \mathrm{s} / \mathrm{m}]$ & $3.1 \cdot 10^{4}$ \\
\hline
\end{tabular}

\subsection{Influence of Superelevation Angle on Wheel Load Reduction}

As analysed in Section 2.2, the SADs of the locomotive components on transition curves have an effect on the distributions of the vertical loads in the suspension system. According to the load transfer characteristics, the asymmetry will also be introduced to the dynamic wheel loads. With or without considering the SAD, the wheelrail vertical forces simulated by the locomotive-track coupled model are given in Fig. 8. In this figure, $F_{z L 1}$ and $F_{z R 1}$ are the outer and inner wheel-rail vertical forces. It can be seen that the wheel-rail vertical forces on the entry and exit transition curves appear to be almost symmetrical about that on the circular curve (Fig. 8a) if the influence of SAD is not taken into account. In this condition, there is little difference between the values in circular curve and the values at the points of spiral to curve and curve to spiral. In case that the SAD is considered in the calculation process, the maximum and
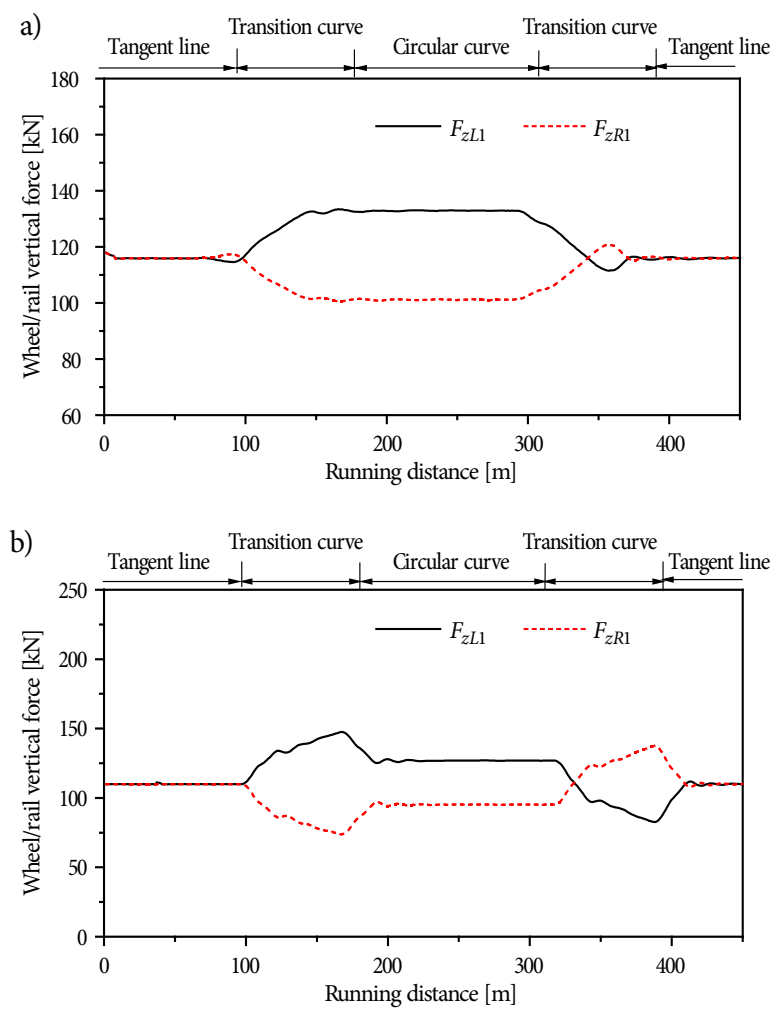

Fig. 8. Wheel-rail vertical forces: a - without considering $\mathrm{SAD} ; \mathrm{b}$ - considering SAD

minimum values of the wheel-rail vertical forces can be obtained nearby the points of spiral to circular curve and circular curve to spiral (Fig. 8b).

Actually, the wheel-rail vertical forces are also related to wheel-rail creep state, wheel flange contact, track random irregularities, etc., and the effect of SAD on wheel-rail dynamic forces is more complicated.

\subsection{Influence of Suspension Parameters on Wheel Load Dynamic Characteristics}

For the same suspension parameters and line geometry parameters used above, the SADs of wheelset and bogie frame are the major factors affecting the wheel load dynamic characteristics. In China, a series of HXD locomotives have been developed and are in active service, 
and among them the six-axle locomotive, which equips with the pull-rod axle box, takes up a high proportion. The primary suspension stiffness is mainly provided by the coil spring, which varies within a limited range. For the secondary suspension, there are two types of structural schemes including coil spring and rubber element. The difference of material and structure in the secondary suspension can produce a wide change range of the vertical stiffness. Furthermore, the curve conditions where the locomotive operates are also complex and various. In this section, the vertical stiffness, transition curve length and outer rail superelevation are selected as the study parameters to investigate their influence on the dynamic characteristics of the wheel load. In order to reveal the influences of the studied parameters, the track irregularities are not taken into account.

Taking the outer wheel-rail vertical force of the first wheelset in curves as example, the influences of these parameters are given below. Fig. 9 compares the wheel-rail vertical forces for the different vertical stiffness of the secondary suspension. With the increase of the vertical stiffness, the wheel loads in the circular curves are very close while the larger differences exist at the entry and exit transition curves. A bigger $k_{s z}$ produces a larger fluctuation amplitude of the wheel-rail vertical force.

Influences of the transition curve length and outer rail superelevation on wheel-rail vertical force are shown in Figs 10-11. It is indicated in Fig. 10 that a longer transition curve will decrease the fluctuation of dynamic wheel load. The essence of lengthening the transition curve is to reduce the gradient of superelevation angle variation along the curve length. For a certain distance between bogie centers, a longer transition curve results in a smaller SAD between the car body and bogie frame, which could alleviate the dynamic fluctuation of the wheel-rail vertical force. The reduction of outer rail superelevation can also change the wheel-rail vertical force (Fig. 11). With a deficient superelevation for the locomotive in the curve, the reduction of the superelevation will increase the roll angles of the car body and bogie frame and the twist phenomenon can be improved simultaneously, so that the impacts of SADs on wheelrail vertical force are weakened.

The variations of superelevation and curve radius are the dominating factors, which produce the twist of

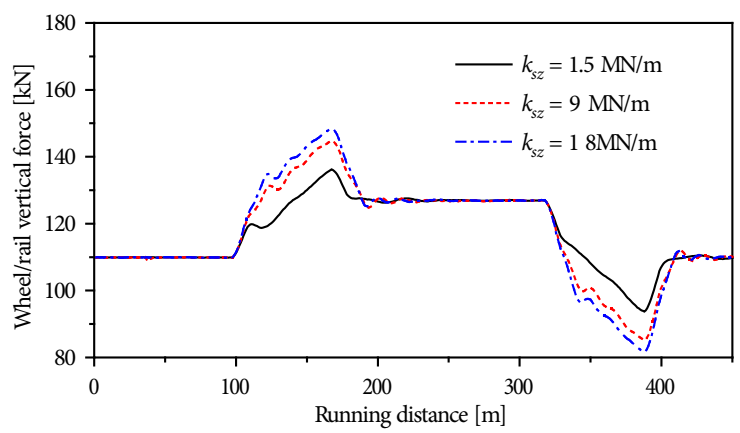

Fig. 9. Comparisons of outer wheel loads in the first wheelset for different secondary suspension stiffness

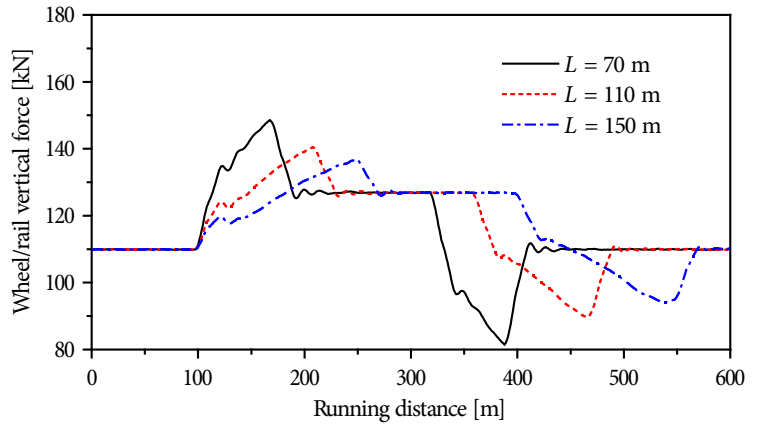

Fig. 10. Comparisons of outer wheel load in the first wheelset for different transition curve length

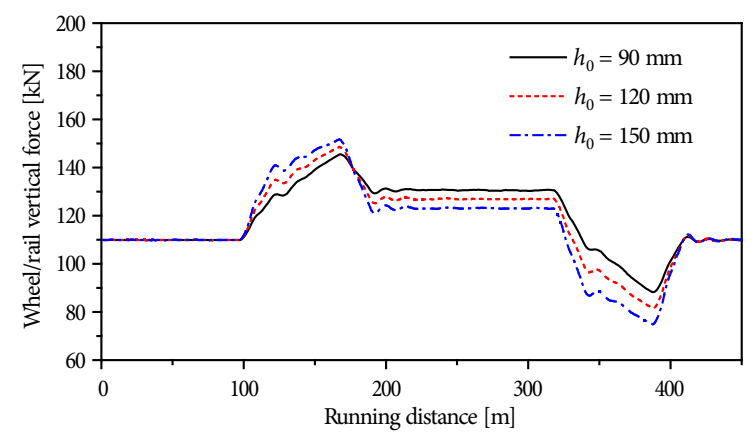

Fig. 11. Comparisons of outer wheel load in the first wheelset for different superelevation

track plane. In order to make the locomotive be able to negotiate a curve smoothly, the twist degree of track should be reduced and the appropriate suspension parameters need to be determined. It should be pointed out that for the vertical wheel-rail forces, a significant difference induced by the track vibration is above the frequencies $>40 \mathrm{~Hz}$ (Zhai et al. 2009). However, the frequencies caused by the curve distortion in transition curve sections are in the low-frequency range far below $40 \mathrm{~Hz}$. Therefore, the influence of track vibration is not obvious in the calculation results.

\section{Field Test on Wheel Load of the Heavy-Haul Locomotive}

In order to investigate the dynamic characteristics of wheel load for the locomotive, a test section was chosen in a curve with the radius of $300 \mathrm{~m}$ in the Chinese Taiyuan-Jiaozuo railway line. The superelevation of outer rail is $120 \mathrm{~mm}$, and the length of transition curve is $70 \mathrm{~m}$. The locomotive running on this line is a $23 \mathrm{t}$ axle load locomotive with $C_{0}-C_{0}$ axle arrangement (Fig. 12). The force sensors and the displacement sensors are installed at primary suspensions of the first and sixth wheelset in vertical direction. Meanwhile, in the vertical direction of the secondary suspension, the displacement sensors are fixed between the car body and bogie frames, as shown in Fig. 13.

The relative displacements of the suspension points reflect the variations of vertical loads in suspension systems. Under the ideal conditions, the loads in the primary suspension of each wheel are equal to $1 / 12$ of the total 

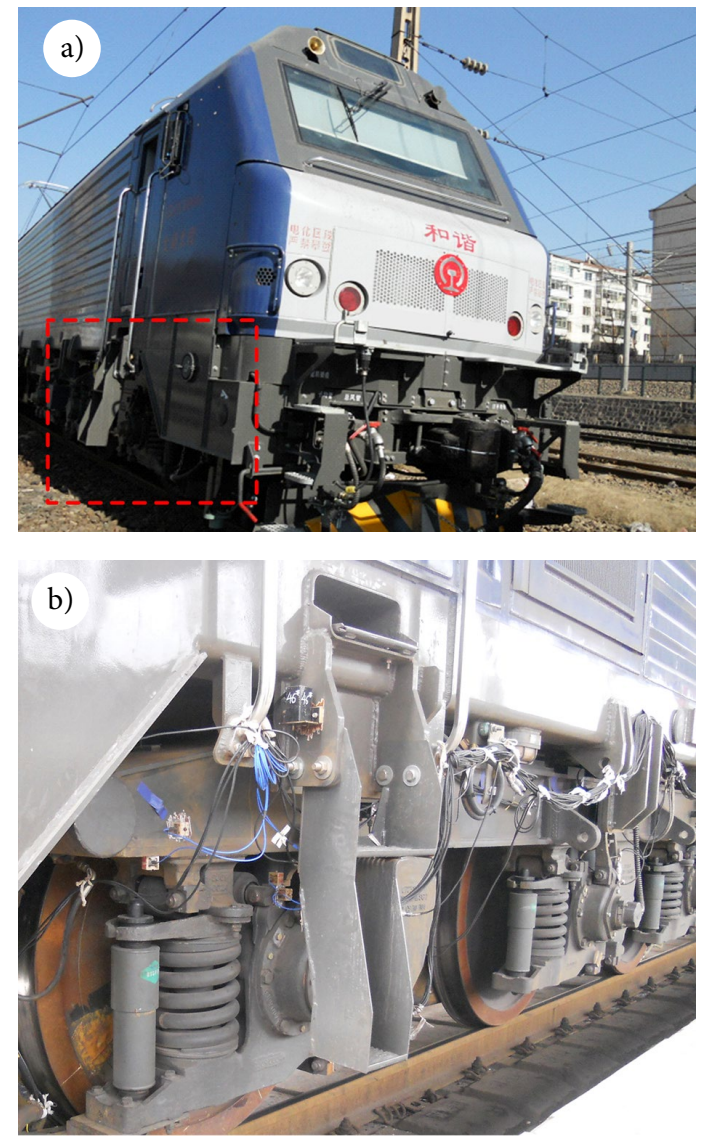

Fig. 12. Onsite test of locomotive dynamics: a - tested locomotive; $\mathrm{b}$ - sensor arrangement

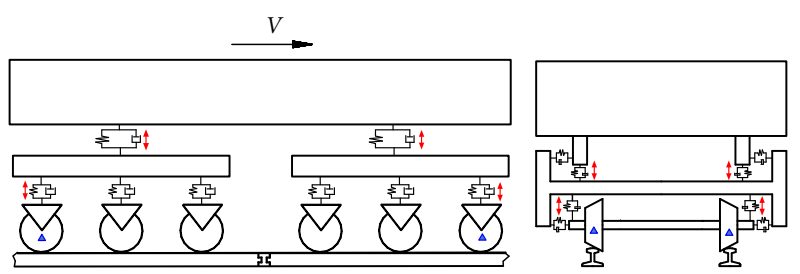

Fig. 13. Testing point arrangements: $\downarrow$ - test point of relative displacement; $\Delta$ - test point of wheel-rail force

weight of the car body and bogie frames. With the compression or stretch of the suspension, the load increases or reduces. For the test results, the positive displacement of suspension system denotes the stretching spring, indicating that the vertical load is decreased. Conversely, the negative value reflects the compressing spring, which means an increase in the vertical load. Fig. 14 shows the actual dynamic displacement of the primary suspension at the first and sixth wheelset. When the locomotive enters and exits the transition curves, the outer displacement in primary suspension becomes from negative to positive, indicating that the suspension works from compressing state to stretching state correspondingly. The dynamic process of load increase and load reduction is distinctly observed. Moreover, the suspension displacements of sixth wheelset vary conversely relative to that of the front wheelset. The dynamic displacements of the secondary suspension are shown in Fig. 15. The measured data agree well with the simulation results in Section 2, verifying the correctness of the theoretical dynamic model.
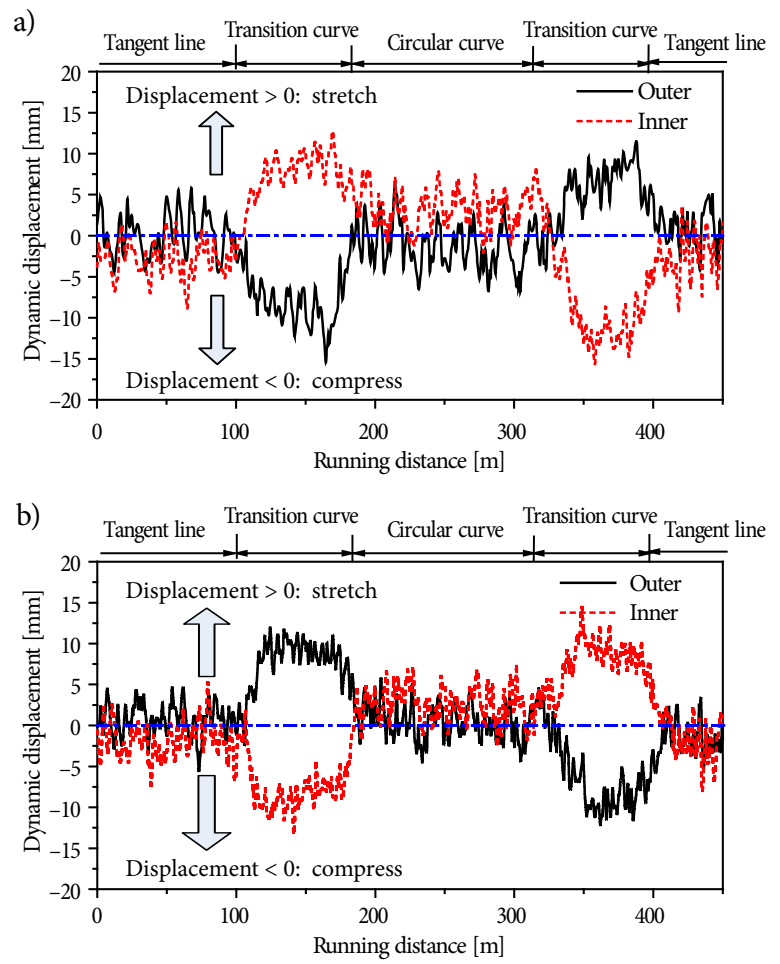

Fig. 14. Measured results of dynamic displacement in primary suspension: $\mathrm{a}$ - the first wheelset; $\mathrm{b}$ - the sixth wheelset
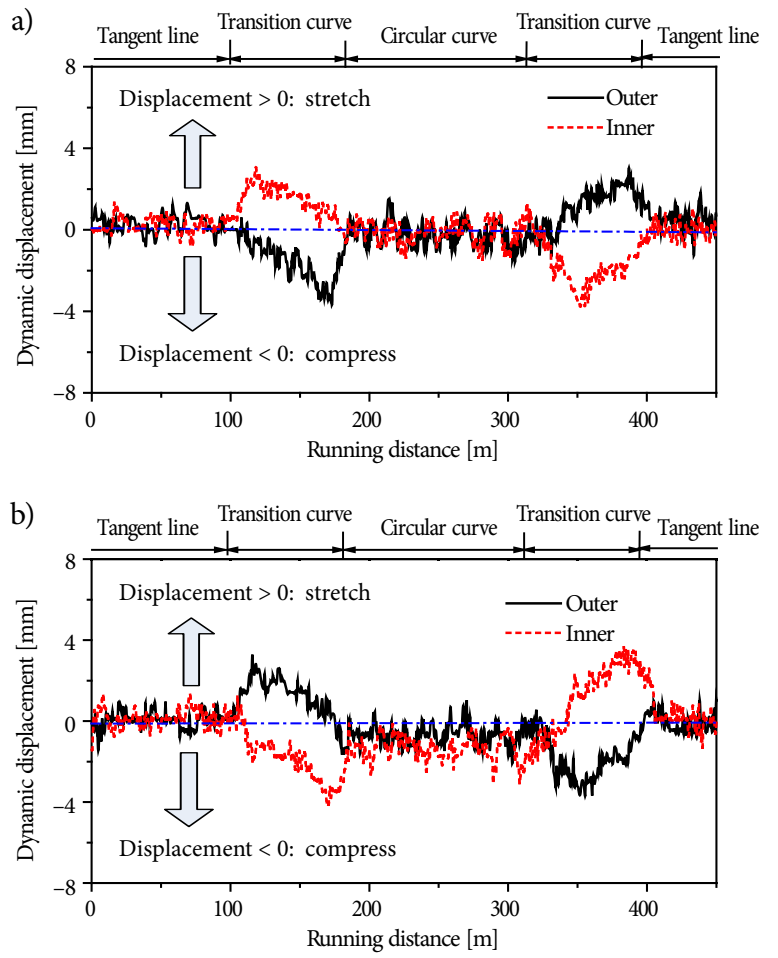

Fig. 15. Measured results of dynamic displacement in secondary suspension: $\mathrm{a}$ - front bogie; $\mathrm{b}$ - rear bogie 
The asymmetric loads in suspension systems determine the dynamic characteristics of wheel load. In order to illustrate the wheel load dynamic property by simulation and field test simultaneously, the wheel-rail forces are also calculated by the locomotive-track coupled model established in Section 3. The input excitation is the random irregularities converted from Chinese mainline spectrum (Zhai et al. 2009), and other operation conditions for simulation keep the same as those of the field test. The measured and simulated wheel-rail vertical forces are compared in Figs 16-17. Under the excitation of track irregularities, the wheel-rail vertical forces fluctuate greatly and their local maximum values partly depend on the track excitation. However, the overall changing trends of these forces are clear. The simulated and measured results indicate that the outer wheel load of the first wheelset reduces at the exit transition curve, and the outer wheel load of the sixth wheelset has a reduction at the entry curve. The simulated and measured results coincide well with each other, verifying the correctness of the proposed theoretical dynamic model.

In summary, the established quasi-static model can reflect the dynamic load characteristics of the suspension system of the locomotive, which negotiates a curved track. The simulated results based on the locomotivetrack coupled dynamic model are closed to that of the field test. In addition, the influence of SADs on wheel load in sections of transition curves should be paid much more attention to. Because if the wheel-rail lateral force does not change, a reduction in wheel load will lead to the increasing in derailment coefficient, which means the running safety of locomotive is deteriorated.
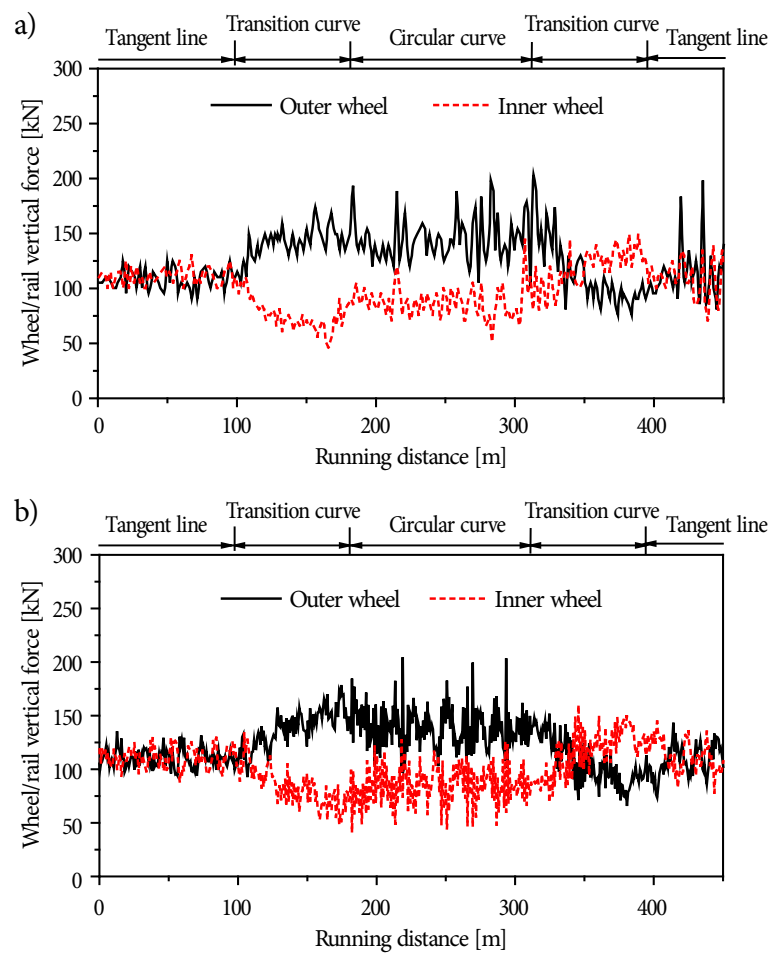

Fig. 16. Comparisons of wheel-rail vertical forces in the first wheelset obtained from test and simulation: $\mathrm{a}$ - measured result; $\mathrm{b}$ - simulated result

\section{Conclusions}

A quasi-static model of the six-axle heavy-haul locomotive is established to investigate the vertical load asymmetry of suspension system in the outer rail side and inner rail side on curve. For analysing the wheel load variations in the same running conditions, the coupled model of locomotive and ballasted track is applied. The simulation results have been validated in a field experiment. The detailed conclusions are drawn as follows:

1) For a locomotive on the curved track, the values of SADs between the bogie frames and car body can significantly affect the load distributions in the secondary suspension system. A plus SAD may lead to the load increase of outer suspension, while a minus $\mathrm{SAD}$ results in the load reduction. From the entry to the exit transition curves, the SAD value of a certain bogie varies between positive and negative, which causes the inverse load transfer characteristics.

2) The changing rules of wheel load and primary suspension force in the vertical direction on a curve are mainly determined by the SADs between the bogie frames and wheelsets as well as them between the car body and bogie frames. The wheel loads in the first and sixth wheelsets are affected most obviously by the SADs.

3) Both of the track geometry and locomotive suspension parameters have significant impact on the load distributions in the heavy-haul locomotive system, and they should be considered synthetically for improving the locomotive dynamic performance.
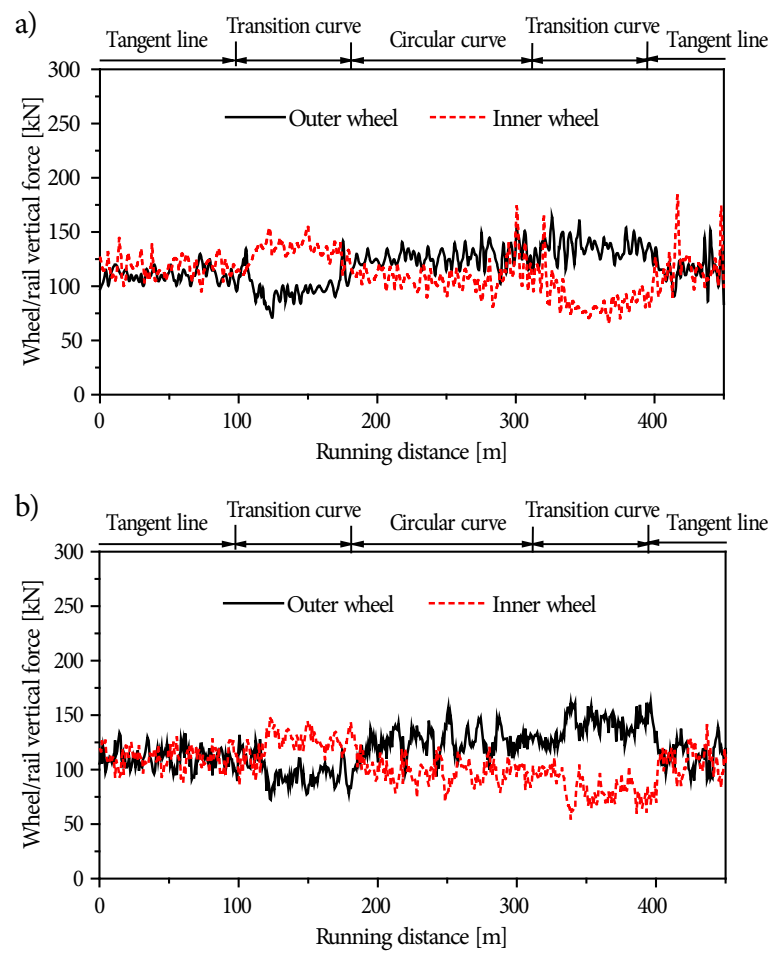

Fig. 17. Comparisons of wheel-rail vertical forces in the sixth wheelset obtained from test and simulation: $\mathrm{a}$ - measured result; $\mathrm{b}$ - simulated result 


\section{Acknowledgements}

This work was supported by the Scientific Research and Development Program of China Railway Corporation under grant 2013J012-A, the National Science and Technology Pillar Plan Program (2013BAG20B00), Science and Technology Research Project of Hebei Province (BJ2016047) and the Scientific Discipline Development Project of Southwest Jiaotong University (2012).

The authors appreciate the great support from China Academy of Railway Sciences for carrying out the field test of the heavy-haul locomotive on a curve.

\section{References}

AS/RISSB 7509.1:2009. Railway Rolling Stock - Dynamic Behaviour - Locomotive Rolling Stock.

BS EN 14363:2005. Railway Applications. Testing for the Acceptance of Running Characteristics of Railway Vehicles. Testing of Running Behavior and Stationary Tests.

Eom, B.-G.; Lee, H. S. 2010. Assessment of running safety of railway vehicles using multibody dynamics, International Journal of Precision Engineering and Manufacturing 11(2): 315-320. http://doi.org/10.1007/s12541-010-0036-x

Gailiené, I. 2012. Investigation into the calculation of superelevation defects on conventional rail lines, Transport 27(3): 229-236. http://doi.org/10.3846/16484142.2012.719198

GM/RT 2141:2000. Resistance of Railway Vehicles to Derailment and Roll-Over.

Gu, A. J. 2007. Railway Track. Beijing: China Railway Publishing House. (in Chinese).

Lipičnik, M. 1998. New form of road/railway transition curve, Journal of Transportation Engineering 124(6): 546-556.

http://dx.doi.org/10.1061/(ASCE)0733-947X(1998)124:6(546)

Long, X.-Y.; Wei, Q.-C.; Zheng, F.-Y. 2010. Dynamic analysis of railway transition curves, Proceedings of the Institution of Mechanical Engineers, Part F: Journal of Rail and Rapid Transit 224(1): 1-14. http://doi.org/10.1243/09544097JRRT287

Miyagaki, K.; Adachi, M.; Sato, Y. 2004. Analytical study on effects of form in transition curve, Vehicle System Dynamics (Supplement): Dynamics of Vehicles on Roads and on Tracks: Proceedings of the 18th IAVSD Symposium, 24-30 August 2003, Kanagawa, Japan, 657-666.

Polach, O.; Berg, M.; Iwnicki, S. 2006. Simulation, in S. Iwnicki (Ed.). Handbook of Railway Vehicle Dynamics, 359-422.

Sinokrot, T.; Nakhaeinejad, M.; Shabana, A. A. 2008. A velocity transformation method for the nonlinear dynamic simulation of railroad vehicle systems, Nonlinear Dynamics 51(1): 289-307. http://doi.org/10.1007/s11071-007-9211-8

Slivsgaard, E. C. 1995. On the Interaction between Wheels and Rails in Railway Dynamics: PhD Thesis. Technical University of Denmark. 196 p. Available from Internet: http://www2.imm.dtu.dk/pubdb/views/edoc_download. php/2103/pdf/imm2103.pdf

Suarez, B.; Mera, J. M.; Martinez, M. L.; Chover, J. A. 2013. Assessment of the influence of the elastic properties of rail vehicle suspensions on safety, ride quality and track fatigue, Vehicle System Dynamics: International Journal of Vehicle Mechanics and Mobility 51(2): 280-300.

http://doi.org/10.1080/00423114.2012.725852
Um, J. H.; Choi, I. Y.; Yang, S. C.; Kim, M. C. 2011. Optimization of alignment considering ride comfort for superimposition of vertical and horizontal curves, Proceedings of the Institution of Mechanical Engineers, Part F: Journal of Rail and Rapid Transit 225(6): 649-662.

http://doi.org/10.1177/0954409710397641

Wang, K. Y.; Zhai, W. M. 2003. Calculation of displacements of vehicle suspension on tangent and curved tracks, Journal of Southwest Jiaotong University 38(2): 122-126. (in Chinese).

Wilson, N.; Fries, R.; Witte, M.; Haigermoser, A.; Wrang, M.; Evans, J.; Orlova, A. 2011. Assessment of safety against derailment using simulations and vehicle acceptance tests: a worldwide comparison of state-of-the-art assessment methods, Vehicle System Dynamics: International Journal of Vehicle Mechanics and Mobility 49(7): 1113-1157. http://doi.org/10.1080/00423114.2011.586706

Zhai, W. M.; Cai, C. B.; Guo, S. Z. 1996. Coupling model of vertical and lateral vehicle/track interactions, Vehicle System Dynamics: International Journal of Vehicle Mechanics and Mobility 26(1): 61-79. http://doi.org/10.1080/00423119608969302

Zhai, W.; Wang, K.; Cai, C. 2009. Fundamentals of vehicletrack coupled dynamics, Vehicle System Dynamics: International Journal of Vehicle Mechanics and Mobility 47(11): 1349-1376. http://doi.org/10.1080/00423110802621561

Zhai, W.; Xia, H; Cai, C.; Gao, M.; Li, X.; Guo, X.; Zhang, N.; Wang, K. 2013. High-speed train-track-bridge dynamic interactions - Part I: theoretical model and numerical simulation, International Journal of Rail Transportation 1(1-2): 3-24. http://doi.org/10.1080/23248378.2013.791498

Zhang, J. Q.; Huang, Y. H.; Li, F. 2010. Influence of transition curves on dynamics performance of railway vehicle, Journal of Traffic and Transportation Engineering 10(4): 39-44. (in Chinese). 